\title{
Double-framed Soft BF-algebras
}

\author{
A. R. Hadipour* \\ Department of Mathematics, Rafsanjan Branch, Islamic Azad University, Rafsanjan, Iran; ar.hadipour@yahoo.com
}

\begin{abstract}
In this paper, we introduce the notion of double-framed soft BF-algebras and study related properties. We consider characterizations of double-framed soft BF-algebras and deal with the product and int-uni structure of double-framed soft BF-algebras.
\end{abstract}

Keywords: Double-Framed Soft BF-algebra, Double-Framed Soft Int-uni, Double-framed Soft Product

\section{Introduction}

We cannot always use classical methods to solve complicated problems in economics, engineering, sociology, medical science and many other fields, because the uncertainties appearing in these domains. There exist four theories: Probability, Fuzzy Set Theory, Interval valued Mathematics and Rough Set Theory, which can considered as mathematical tools for dealing with imperfect knowledge. All these tools need the pre-specification of some parameter to start with, e.g. probability density function in probability, membership function in the Fuzzy set theory and equivalence relation in the tough set theory. This requirement, seen in the backdrop of imperfect or incomplete knowledge, raises some problems. Molodtsov ${ }^{1}$ introduced the notion of soft set to deal with problems of incomplete information. Soft Set Theory does not need the specification of a parameter. This makes soft set theory a natural mathematical tool for approximate reasoning. Later, Maji et al..$^{-4}$ have further studied the theory of soft set and by using it solve some decision making problems. In 2001, Maji et al. ${ }^{4}$ introduced the notion of fuzzy soft set, which is a combination of fuzzy set and soft set and studied its properties in details.

In 1966, Imai and Iseki ${ }^{5,6}$ introduced two classes of algebras: BCK-algebras, BCI-algebras and the class of BCK-algebras is a proper subclass of the class of BCI-algebras. J. Neggers and H. S. Kim introduced the not-ion of B-algebras as a generalization of BCK-algebra ${ }^{7}$
Recently, A. Walendziak introduced the notion of a BF-algebra ${ }^{8}$.

In this paper, we introduced double-framed soft BF-algebras and investigate related properties. Finally we characterize double-framed soft BF-algebras and deal with the product and int-uni structure of double-framed soft BF-algebras.

\section{Preliminaries}

In this section, we cite the fundamental definitions that will be used in the sequel:

Definition 2.1 Let $X$ be a non-empty set, * be a binary operation and a constant 0 . Then $(\mathrm{X}, *, 0)$ is called a BF-algebra if satisfied the following axioms:

(BF1) $x * x=0$

(BF2) $x * 0=x$,

(BF3) $0 *(x * y)=(y * x)$,

for all $x, y \in X$.

EXAMPLE 2.2 (a) Let $\mathrm{R}$ be the set of real numbers and $A=(\mathrm{R} ; *, 0)$ be the algebra with the operation $*$ defined $^{8}$ by

$$
x * y= \begin{cases}x & \text { if } y=0 \\ y & \text { if } x=0 \\ 0 & \text { otherwise }\end{cases}
$$


Then $\mathrm{A}$ is a $\mathrm{BF}$-algebra.

(b) Let $A=[0 ; \infty)$. Define the binary operation $*$ on $\mathrm{A}$ as follows: $x * y=|x-y|$, for all $x, y \in A$. Then $(A ; *, 0)$ is a BF-algebra.

Proposition 2.3 Let $\mathrm{X}$ be a BF-algebra. Then for any $\mathrm{x}$ and $\mathrm{y}$ in $\mathrm{X}$, the following hold ${ }^{8}$ :

(a) $0 *(0 * x)=x$ for all $x \in A$;

(b) if $0 * x=0 * y$, then $\mathrm{x}=\mathrm{y}$ for any $x, y \in A$;

(c) if $x * y=0$, then $y * x=0$ for any $x, y \in A$.

Definition 2.4 ${ }^{8} \mathrm{~A}$ non-empty subset $\mathrm{S}$ of a BF-algebra $\mathrm{X}$ is called a sub algebra of $\mathrm{X}$ if $x * y \in S$ for any $x, y \in S$.

DeFinition 2.5 A mapping $g: X \rightarrow Y$ of BF-algebras is called a BF-homomorphism if $g(x * y)=g(x) * g(y)$, for all $x, y \in x$.

Let $\mathrm{X}$ be a set. A fuzzy set $\mathrm{A}$ in $\mathrm{X}$ is determined with a membership function $\mu_{A}: X \rightarrow[0,1]$. Let $f$ be a mapping from the set $\mathrm{X}$ to the set $\mathrm{Y}$ and let $\mathrm{B}$ be a fuzzy set in $\mathrm{Y}$ with membership function $\mu_{B}$.

The inverse image of $\mathrm{B}$, denoted by $f^{-1}(B)$, is the fuzzy set in $\mathrm{X}$ with membership function $\mu_{f^{-1}(B)}$ defined by $\mu_{f^{-1}(B)}(x)=\mu_{B}(f(x))$ for all $x \in X$.

Conversely, let A be a fuzzy set in X with membership function $\mu_{A}$ Then the image of $\mathrm{A}$, denoted by $f(A)$, is the fuzzy set in Y such that:

$\mu_{f(A)}(y)=\left\{\begin{array}{cc}\sup _{z \in f^{-1}(y)} \mu_{A}(z) & \text { if } f^{-1}(y)=\{x: f(x)=y\} \neq \varnothing, \\ 0 & \text { otherwise }\end{array}\right.$

DeFinition 2.6 Let $f$ be a fuzzy set of $X$. Then $f$ is called a fuzzy sub algebra of $X$ if it satisfies

$(>=) f(x * y) \neq \min \{f(x), f(y)\}$, whenever $x, y \in X$.

Molodtsov $^{1}$ defined the notion of soft set by: let $U$ be an initial universe set and $E$ be a set of parameters also $P(U)$ denotes the power set of $U$ and $A \subseteq E$.

Definition 2.7 A pair $(F, A)$ is called a soft set over $U$ whenever $F$ is a mapping given by $F: A \rightarrow P(U)$.

By a soft set over $U$, we mean that a parameterized family of subsets of a universe $U$. For $x \in A, F(x)$ can be considered as a set of $x$-elements of the soft set $(F, A)$, or as the set of $x$-approximate elements of the soft set $(F, A)$. Therefore a soft set is not a set, for illustration, Molodtsov constructed several examples ${ }^{1}$.
DEFINITION 2.8 Let $U$ be an initial universe set and $E$ be a set of parameters. Let $F(U)$ denote the set of all fuzzy sets in $U$. Then $(F, A)$ is called a fuzzy soft set over $U$ in which $A \subset E$ and $F$ is a map given ${ }^{8}$ by

$$
F: A \rightarrow P(U) \text {. }
$$

In general, for every $x \in A, F[x]$ is a fuzzy set in $U$ and it is called fuzzy value set of parameter $x$. If for every $x \in A$, $F[x]$ is a crisp subset of $U$, then $(F, A)$ is degenerated to be the standard soft set. Thus, from the above definition, it is clear that fuzzy soft sets are a generalization of standard soft sets.

Definition 2.9 Let $(F, A)$ and $(G, B)$ be two fuzzy soft sets over a common universe $U$. The union of $(F, A)$ and $(G, B)$ is defined to be the fuzzy soft set $(H, C)$ satisfying the following conditions ${ }^{5}$ :

(i) $C=A \cup B$,

(ii) for all $e \in C$,

$$
H[e]=\left\{\begin{array}{c}
F[e] \quad \text { if } e \in A \backslash B, \\
G[e] \quad \text { if } e \in B \backslash A, \\
F[e] \cup G[e] \text { if } e \in A \cap B .
\end{array}\right.
$$

In this case, we write $(F, A) \cup(G, B)=(H, C)$.

Definition 2.10 If $(F, A)$ and $(G, B)$ are two fuzzy soft sets over a common universe $U$, then " $(F, A) \operatorname{AND}(G, B)$ " denoted by $(F, A)^{\wedge}(G, B)$ is defined by $(F, A)^{\wedge}(G, B)=$ $(\mathrm{H}, \mathrm{A} \times \mathrm{B})$ where $H[x, y]=F[x] \cap G[y]$ for all $(x, y)$ $\in A \times B^{5}$.

Throughout this subsection $U$ refers to an initial universe, $E$ is a set of parameters; $S$ and $T$ are two BF-algebras.

DEFINITION 2.11 Let $P(S)$ be the power set of $S$, a pair (F, A) is called a soft set over $S$ where $\mathrm{F}$ is a mapping given ${ }^{1}$ by

$$
F: \mathrm{A} \rightarrow P(S)
$$

Definition 2.12 Let (F, A) be a soft set over $S$, (F, A) is said to be a soft BF-algebra over $S$ if and only if $F(x)$ is a sub algebra of $\mathrm{S}$, for all $x \in \mathrm{A}^{10}$.

Definition 2.13 A pair $(\Lambda, \Sigma)$ is called a fuzzy soft set over $S$, where $\Lambda: \Sigma \rightarrow \tilde{P}(S)$ is a mapping, $\tilde{P}(S)$ being the set of all fuzzy sets of $S^{4}$.

Definition 2.14 Let $(\Lambda, \Sigma)$ and $(\Delta, \Omega)$ be two fuzzy soft sets over $S,(\Lambda, \Sigma)$ is called a fuzzy soft subset of $(\Delta, \Omega)$, denoted by $(\Lambda, \Sigma) \tilde{\subseteq}(\Delta, \Omega)^{4}$, if 
(i) $\Sigma \subseteq \Omega$, (ii) for each $\varepsilon \in \Sigma, \Lambda(\varepsilon) \leq \Delta(\varepsilon)$.

Definition $2.15^{9}$ Let $(\Lambda, \Sigma)$ and $(\Delta, \Omega)$ be two fuzzy soft sets over $S$ with $\Sigma \cap \Omega \neq \varnothing$.The intersection of them, denoted by $(\Lambda, \Sigma) \tilde{\wedge}(\Delta, \Omega)=(\Theta, \Xi)$, is a fuzzy soft set over $S$, where $\Xi=\Sigma \bigcap \Omega$, and for each $\varepsilon \in \Xi, \Theta(\varepsilon)=\Lambda(\varepsilon) \wedge \Delta(\varepsilon)$. Where $\Lambda(\varepsilon) \wedge \Delta(\varepsilon)$ means the intersection of fuzzy subsets $\Lambda(\varepsilon)$ and $\Delta(\varepsilon)$.

DeFinition 2.169 The union of two fuzzy soft sets $(\Lambda, \Sigma)$ and $(\Delta, \Omega)$ over $S$, denoted by $(\Lambda, \Sigma) \tilde{v}(\Delta, \Omega)=(\Theta, \Xi)$, is a fuzzy soft set over $S$, where $\Xi=\Sigma \cup \Omega$, and for each

$\varepsilon \in \Xi$

$$
\Theta(\varepsilon)=\left\{\begin{array}{rll}
\Lambda(\varepsilon), & \text { if } & \varepsilon \in \Sigma-\Omega, \\
\Delta(\varepsilon), & \text { if } & \varepsilon \in \Omega-\Sigma, \\
\Lambda(\varepsilon) \vee \Delta(\varepsilon), & \text { if } & \varepsilon \in \Sigma \bigcap \Omega .
\end{array}\right.
$$

where $\Lambda(\varepsilon) \vee \Delta(\varepsilon)$ means the union of fuzzy subsets $\Lambda(\varepsilon)$ and $\Delta(\varepsilon)$.

Definition 2.17 Let $U$ be a universe and $E$ a set of parameters. Collection of all fuzzy soft sets over $U$ with parameters from $E$, is called a fuzzy soft class and denoted as $(U, E)^{4}$.

Definition $2.18^{10}$ Let $(X, E)$ and $\left(Y, E^{\prime}\right)$ be classes of fuzzy soft sets over $X$ and $Y$ with parameters from $E$ and $E^{\prime}$, respectively. Let $u: X \rightarrow Y$ and $p: E \rightarrow E^{\prime}$ be two mappings. For a fuzzy soft set $(\Lambda, \Sigma)$ in $(X, E)$, where $\Sigma \subseteq E$, the image of $(\Lambda, \Sigma)$ under the fuzzy soft function $f=(u, p)$, denoted by $f(\Lambda, \Sigma)$, is the fuzzy soft set over $Y$ defined by $f(\Lambda, \Sigma)=(u(\Lambda), p(\Sigma))$, where

$$
\begin{aligned}
& u(\Lambda)(\beta)(y)= \\
& \begin{cases}\underset{x \in u^{-1}(y) \alpha \in p^{-1}(\beta) \cap \Sigma}{\vee} \Lambda(\alpha)(x), & \text { if } u^{-1}(y) \neq \varnothing, \text { otherwise } \\
0 & \forall \beta \in p(\Sigma), \forall y \in Y .\end{cases}
\end{aligned}
$$

Definition 2.19 ${ }^{10}$ Let $(U, E)$ and $\left(Y, E^{\prime}\right)$ be classes of fuzzy soft sets over $X$ and $Y$ with parameters from $E$ and $E^{\prime}$, respectively. Let $u: X \rightarrow Y$ and $p: E \rightarrow E^{\prime}$ be two mappings, $(\Delta, \Omega)$ be a fuzzy soft set in $\left(Y, E^{\prime}\right)$, where $\Omega \subseteq E^{\prime}$. The inverse image of $(\Delta, \Omega)$ under the fuzzy soft function $f=(u, p)$, denoted by $f^{-1}(\Delta, \Omega)$, is the fuzzy soft set over $X$ defined by $f^{-1}(\Delta, \Omega)=\left(u^{-1}(\Delta), p^{-1}(\Omega)\right)$, where

$u^{-1}(\Delta)(\alpha)(x)=\Delta(p(\alpha))(u(x)), \quad \forall \alpha \in p^{-1}(\Omega), \forall x \in X$.
Definition 2.20 A double-framed pair $(\langle\alpha, \beta\rangle, A)$ is called a double-framed soft set over $U$, where $\alpha$ and $\beta$ are mappings from A to $P(U)^{10}$.

\section{Double-framed Soft BF-algebra}

In what follows, we take $E=X$ as a set of parameters, which is a BF-algebra and $A, B, C, \ldots$ as sub algebras of $E$ unless otherwise specified.

Definition 3.1 A double-framed soft set $(\langle\alpha, \beta\rangle, A)$ over $U$ is called a double-framed soft algebra over $U$ if it satisfies

$$
\begin{aligned}
& \alpha(x * y) \supseteq \alpha(x) \cap \alpha(y), \\
& \beta(x * y) \subseteq \beta(x) \cup \beta(y)
\end{aligned}
$$

for all $x, y \in A$.

EXAMPLE 3.2 Suppose that there are five houses in the initial universe set $U$ given by

$$
U=\left\{h_{1}, h_{2}, h_{3}\right\}
$$

Let a set of parameters $E=\left\{e_{1}, e_{2}, e_{3}\right\}$ be a set of status of houses in which

$e_{1}$ stands for the parameter "beautiful," $e_{2}$ stands for the parameter "cheap," $e_{3}$ stands for the parameter "in good location," with the following binary operation:

\begin{tabular}{|l|l|l|l|}
\hline$*$ & $e_{1}$ & $e_{2}$ & $e_{3}$ \\
\hline$e_{1}$ & $e_{1}$ & $e_{2}$ & $e_{3}$ \\
\hline$e_{2}$ & $e_{2}$ & $e_{1}$ & $e_{1}$ \\
\hline$e_{3}$ & $e_{3}$ & $e_{1}$ & $e_{1}$ \\
\hline
\end{tabular}

Then $\left(E, *, e_{1}\right)$ is a BF-algebra. For a sub algebra $A=$ $\left\{e_{1}, e_{2}\right\}$ of $\mathrm{E}$, consider a double framed soft set $\left.(<\alpha, \beta\rangle, A\right)$ over $U$ as follows:

$$
\begin{gathered}
\alpha: A \rightarrow P(U) \\
\alpha(x)=\left\{\begin{array}{l}
\left\{h_{1}, h_{2}, h_{3}\right\} \text { if } x=e_{1} \\
\left\{h_{1}, h_{2}\right\} \text { if } x=e_{2}
\end{array}\right.
\end{gathered}
$$

and

$$
\begin{gathered}
\beta: A \rightarrow P(U) \\
\beta(x)=\left\{\begin{array}{lll}
\left\{h_{1}\right\} & \text { if } & x=e_{1} \\
\left\{h_{1}, h_{2}\right\} & \text { if } x=e_{2}
\end{array}\right.
\end{gathered}
$$


It is routine to verify that $(\langle\alpha, \beta\rangle, A)$ is a double-framed soft algebra over $U$.

LEмма 3.3 Every double-framed soft algebra $(<\alpha, \beta\rangle, A)$ over $U$ satisfies the following condition:

$$
\alpha(0) \supseteq \alpha(x), \beta(0) \subseteq \beta(x)
$$

for all $x \in A$.

Proof. It is straightforward.

Proposition 3.4 For a double-framed soft algebra $(<\alpha, \beta>, A)$ over $U$, the following are equivalent:

(i) $\alpha(0)=\alpha(x), \beta(0)=\beta(x)$, for all $x \in A$,

(ii) $\alpha(y) \subseteq \alpha(x * y) \beta(y) \supseteq \beta(x * y)$,

for all $x, y \in A$.

Proof. Let $\alpha(0)=\alpha(x), \beta(0)=\beta(x)$ for all $x \in A$. We have

$$
\begin{aligned}
& \alpha(y)=\alpha(0) \cap \alpha(y)=\alpha(x) \cap \alpha(y) \subseteq \alpha(x * y) \\
& \beta(y)=\beta(0) \cup \beta(y)=\beta(x) \cup \beta(y) \supseteq \beta(x * y)
\end{aligned}
$$

for all $x, y \in A$.

Conversely, let $\alpha(y) \subseteq \alpha(x * y) \quad \beta(y) \supseteq \beta(x * y)$, for all $x, y \in A$. it is enough to put $y=0$ in it, then we have $\alpha(0) \subseteq \alpha(x)$ and by the above lemma we have $\alpha(0)=\alpha(x)$, for all $x \in A$.

Proposition 3.5 In a BF-algebra $\mathrm{X}$, every doubleframed soft algebra $(\langle\alpha, \beta\rangle, A)$ over $\mathrm{U}$ satisfies the following condition:

$$
\begin{aligned}
& \alpha(x *(0 * y)) \supseteq \alpha(x) \cap \alpha(y) \\
& \beta(x *(0 * y)) \subseteq \beta(x) \cup \beta(y)
\end{aligned}
$$

for all $x, y \in A$.

Proof. By Lemma 3.3, we have

$\alpha(x *(0 * y)) \supseteq \alpha(x) \cap \alpha(0 * y) \supseteq \alpha(x) \cap \alpha(0) \cap \alpha(y)=\alpha(x) \cap \alpha(y)$ $\beta(x *(0 * y)) \subseteq \beta(x) \cup \beta(0 * y) \subseteq \beta(x) \cup \beta(0) \cup \beta(y)=\beta(x) \cup \beta(y)$

for all $x, y \in A$.

Let $(<\alpha, \beta>$, A) and $(<\delta, \varepsilon\rangle, B)$ be double-framed soft sets over a common universe $\mathrm{U}$. Then $(\langle\alpha, \beta\rangle, A)$ is called a double-framed soft subset of $(\langle\delta, \varepsilon\rangle, B)$, denoted by $(<\alpha, \beta>, A) \Subset(<\delta, \varepsilon>, B)$, if (i) $A \subseteq B$,

(ii) $\alpha(e)$ and $\delta(e)$ are identical approximations also $\beta(e)$ and $\varepsilon(e)$ are identical approximations, for all $e \in A$.

Theorem 3.6 Let $(\langle\alpha, \beta\rangle, A)$ be a double-framed soft subset of a double-framed soft set $(\langle\delta, \varepsilon\rangle, B)$. If $(<\delta, \varepsilon>, B)$ is a double-framed soft algebra over $\mathrm{U}$, then so is $(<\alpha, \beta>, A)$.

Proof. Let $x, y \in A$. Then $x, y \in B$ therefore we have

$$
\begin{aligned}
& \alpha(x) \cap \alpha(y)=\delta(x) \cap \delta(y) \subseteq \delta(x * y)=\alpha(x * y) \\
& \beta(x) \cup \beta(y)=\varepsilon(x) \cup \varepsilon(y) \supseteq \varepsilon(x * y)=\beta(x * y)
\end{aligned}
$$

Hence $(\langle\alpha, \beta\rangle, A)$ is a double-framed soft algebra over U.

For two double-framed soft sets $(<\alpha, \beta>, A)$ and $(<\delta, \varepsilon>, A)$ over $\mathrm{U}$, the double-framed soft int-uni set of $(<\alpha, \beta\rangle, A)$ and $(\langle\delta, \varepsilon\rangle, A)$ is defined to be a doubleframed soft set

$(<\alpha \tilde{\bigcap} \delta, \tilde{\jmath} \varepsilon>, A)$ where

$$
\begin{aligned}
& \alpha \tilde{\bigcap} \delta: A \rightarrow P(U) \\
& x \mapsto \alpha(x) \cap \delta(x)
\end{aligned}
$$

and

$$
\begin{aligned}
& \beta \tilde{\cup} \varepsilon: A \rightarrow P(U) \\
& x \mapsto \beta(x) \cup \varepsilon(x)
\end{aligned}
$$

It is denoted by $(<\alpha, \beta>, A) \Pi(<\delta, \varepsilon>, A)=(<\alpha \tilde{\bigcap} \delta$, $\beta \tilde{\bigcup} \varepsilon>, A)$

Theorem 3.7 The double-framed soft int-uni set of two double-framed soft algebras $(<\alpha, \beta\rangle, A)$ and $(<\delta, \varepsilon\rangle, A)$ over $\mathrm{U}$ is a double-framed soft algebra over $\mathrm{U}$.

Proof. For any $x, y \in A$, we have

$$
\begin{aligned}
& (\alpha \tilde{\bigcap} \delta)(x * y)=\alpha(x * y) \cap \delta(x * y) \\
& \supseteq(\alpha(x) \cap \alpha(y)) \cap(\delta(x) \cap \delta(y)) \\
& =(\alpha(x) \cap \delta(x)) \cap(\alpha(y) \cap \alpha(y)) \\
& =(\alpha \tilde{\bigcap} \delta)(x) \cap(\alpha \tilde{\bigcap} \delta)(y) \\
& (\tilde{\cup} \varepsilon)(x * y)=\beta(x * y) \cup \varepsilon(x * y) \\
& \subseteq(\beta(x) \cup \beta(y)) \cup(\varepsilon(x) \cup \varepsilon(y)) \\
& =(\beta(x) \cup \varepsilon(x)) \cup(\beta(y) \cup \varepsilon(y)) \\
& =(\tilde{\cup} \varepsilon)(x) \cup(\tilde{\jmath} \varepsilon)(y)
\end{aligned}
$$


Therefore $\quad(<\alpha, \beta>, A) \Pi(<\delta, \varepsilon>, A)$ is a doubleframed soft algebra over $\mathrm{U}$.

For a double-framed soft set $(\langle\alpha, \beta\rangle, A)$ over $\mathrm{U}$ and two subsets $R$ and $S$ of $\mathrm{U}$, the $R$ - inclusive set and the $S$-exclusive set of $(\langle\alpha, \beta\rangle, A)$, denoted by $i_{A}(\alpha, R)$ and $e_{A}(\beta, S)$ respectively, are defined as follows:

$$
\begin{aligned}
& i_{A}(\alpha, R):=\{x \in A \mid R \subseteq \alpha(x)\} \\
& e_{A}(\beta, S):=\{x \in A \mid S \supseteq \beta(x)\}
\end{aligned}
$$

respectively. The set $D F_{A}(\alpha, \beta)_{(R, S)}:=\{x \in A \mid R \subseteq \alpha(x), S \supseteq$ $\beta(x)\}$ is called a double-framed including set of $(<\alpha, \beta>, A)$. It is clear that

$$
D F_{A}(\alpha, \beta)_{(R, S)}=i_{A}(\alpha, R) \cap e_{A}(\beta, S) .
$$

TheOREM 3.8 For a double-framed soft set $(\langle\alpha, \beta\rangle, E)$ over $\mathrm{U}$, the following are equivalent:

(i) $(\langle\alpha, \beta\rangle, E)$ is a double-framed soft algebra over $\mathrm{U}$,

(ii) for every subsets $R$ and $S$ of $U$ with $R \in \operatorname{Im}(\alpha)$ and $S \in \operatorname{Im}(\beta)$, the $R$-inclusive set and the $S$-exclusive set of $(<\alpha, \beta>, E)$ are sub algebras of $\mathrm{E}$.

Proof. Assume that $(\langle\alpha, \beta\rangle, E)$ is a double-framed soft algebra over U. Let $x, y \in E$ be such that $x, y \in i_{E}(\alpha, R)$ and $x, y \in e_{E}(\beta, S)$ for every subsets $R$ and $S$ of $\mathrm{U}$ with $R \in \operatorname{Im}(\alpha)$ and $S \in \operatorname{Im}(\beta)$. Therefore we have

$$
\begin{aligned}
& R \subseteq \alpha(x) \cap \alpha(y) \subseteq \alpha(x * y) \\
& S \supseteq \beta(x) \cup \beta(y) \supseteq \beta(x * y)
\end{aligned}
$$

Hence $x * y \in i_{E}(\alpha, R)$ and $x * y \in e_{E}(\beta, S)$, therefore $i_{E}(\alpha, R)$ and $e_{E}(\beta, S)$ are sub algebras of $\mathrm{E}$.

Conversley, suppose that $i_{E}(\alpha, R)$ and $e_{E}(\beta, S)$ are sub algebras of E. Let $x, y \in E$ be such that $a(x)=R_{x}$, $\alpha(y)=R_{y}, \beta(x)=S_{x}$ and $\beta(y)=S_{y}$. Taking $R=R_{y} \cap R_{x}$ and $S=S_{y} \cap S_{x}$ implies that $x, y \in i_{E}(\alpha, R)$ and $x, y \in e_{E}(\beta, S)$. Hence, $x * y \in i_{E}(\alpha, R)$ and $x * y \in e_{E}(\beta, S)$ which imply that

$$
\begin{aligned}
& \alpha(x) \cap \alpha(y)=R_{x} \cap R_{y}=R \subseteq \alpha(x * y) \\
& \beta(x) \cup \beta(y)=S_{x} \cup S_{y}=S \supseteq \beta(x * y)
\end{aligned}
$$

Therefore $(<\alpha, \beta\rangle, E)$ is a double-framed soft algebra over U.
Corollary 3.9 If $(<\alpha, \beta\rangle, E)$ is a double-framed soft algebra over $\mathrm{U}$, then the double-framed including set of $(<\alpha, \beta\rangle, E)$ is a sub algebra $\mathrm{X}$.

For any double-framed soft set $(\langle\alpha, \beta\rangle, E)$ over $\mathrm{U}$, let $\left.\left(<\alpha^{*}, \beta^{*}\right\rangle, E\right)$ be a double-framed soft set over $\mathrm{U}$ defined by

$$
\begin{gathered}
\alpha^{*}: E \rightarrow P(U) \\
x \rightarrow \begin{cases}\alpha(x) & \text { if } \quad x \in i_{E}(\alpha, R) \\
T \quad \text { otherwise }\end{cases}
\end{gathered}
$$

and

$$
\begin{gathered}
\beta^{*}: E \rightarrow P(U) \\
x \rightarrow \begin{cases}\beta(x) & \text { if } \quad x \in e_{E}(\beta, S) \\
V \quad \text { otherwise }\end{cases}
\end{gathered}
$$

where $R, S, T$ and $V$ are subsets of $\mathrm{U}$ with $T \subset \alpha(x)$ and $V \supset \beta(x)$.

Theorem 3.10 If $(\langle\alpha, \beta\rangle, E)$ is a double-framed soft algebra over $\mathrm{U}$, then so is $\left(\left\langle\alpha^{*}, \beta^{*}\right\rangle, E\right)$.

Proof. Assume that $(\langle\alpha, \beta\rangle, E)$ is a double-framed soft algebra over $\mathrm{U}$. Then $i_{E}(\alpha, R)$ and $e_{E}(\beta, S)$ are sub algebras of $\mathrm{E}$ for every subsets $R$ and $S$ of $U$ with $R \in \operatorname{Im}(\alpha)$ and $S \in \operatorname{Im}(\beta)$.

Let $x, y \in E$ If $x, y \in i_{E}(\alpha, R)$, then $x * y \in i_{E}(\alpha, R)$. Thus,

$$
\begin{aligned}
& \alpha *(x) \cap \alpha *(y)=\alpha(x) \cap \alpha(y) \subseteq \alpha(x * y)=\alpha *(x * y) \\
& \text { If } x \notin i_{E}(\alpha, R) \text { or } y \notin i_{E}(\alpha, R) \text {, then } \alpha *(x)=T \text { or } \\
& \alpha *(y)=T \text {. Hence, }
\end{aligned}
$$

$$
\alpha *(x) \cap \alpha *(y)=T \subseteq \alpha *(x * y) .
$$

Now, if $x, y \in e_{E}(\beta, S)$, then $x * y \in e_{E}(\beta, S)$. Thus,

$$
\beta *(y) \cup \beta *(y)=\beta(x) \cup \beta(y) \supseteq \beta(x * y)=\beta *(x * y)
$$

If $x \notin e_{E}(\beta, S)$ or $y \notin e_{E}(\beta, S)$, then $\beta *(x)=V$ or $\beta^{*}(y)=V$. Hence,

$$
\beta^{*}(x) \cup \beta^{*}(y)=V \supseteq \beta^{*}(x * y) .
$$

Therefore, $\left(\left\langle\alpha^{*}, \beta^{*}\right\rangle, E\right)$ is a double-framed soft algebra over $\mathrm{U}$.

Let $(<\alpha, \beta>, A)$ and $(<\alpha, \beta>, B)$ be double-framed soft sets over $\mathrm{U}$. The $\left(\alpha_{\wedge}, \beta_{\mathrm{v}}\right)$-product 
of $(<\alpha, \beta>, A)$ and $(<\alpha, \beta>, B)$ is defined to be a double-framed soft set $\left(<\alpha_{A \wedge B}, \beta_{A \wedge B}>, A \times B\right)$ over $\mathrm{U}$ in which

$$
\begin{aligned}
& \alpha_{A \wedge B}: A \times B \rightarrow P(U) \\
& (x, y) \mapsto \alpha(x) \cap \alpha(y) \\
& \beta_{A \wedge B}: A \times B \rightarrow P(U) \\
& (x, y) \mapsto \beta(x) \cup \beta(y)
\end{aligned}
$$

Theorem 3.11 For any BF-algebras $\mathrm{E}$ and $\mathrm{F}$ as sets of parameters, let $(<\alpha, \beta\rangle, E)$ and $(\langle\alpha, \beta\rangle, F)$ be doubleframed soft algebras over $\mathrm{U}$. Then the $\left(\alpha_{\wedge}, \beta_{\mathrm{v}}\right)$-product of $(<\alpha, \beta>, E) \mathrm{E}$ and $(<\alpha, \beta>, F)$ is also a double-framed soft algebra over $\mathrm{U}$.

Proof. Note that $(E \times F, \square,(0,0))$ is a BF-algebra. For any $(x, y),(a, b) \in E \times F$, we have

$$
\begin{aligned}
& \alpha_{E \wedge F}((x, y) \square(a, b))=\alpha_{E \wedge F}(x * a, y * b)=\alpha(x * a) \cap \\
& \alpha(y * b) \supseteq(\alpha(x) \cap \alpha(a)) \cap(\alpha(y) \cap \alpha(b))=(\alpha(x) \cap \\
& \alpha(y)) \cap(\alpha(a) \cap \alpha(b))=\alpha_{E \wedge F}(x, y) \cap \alpha_{E \wedge F}(a, b),
\end{aligned}
$$

and

$$
\begin{aligned}
& \alpha_{E \wedge F}((x, y) \square(a, b))=\alpha_{E \wedge F}(x * a, y * b)=\alpha(x * a) \\
& \cap \alpha(y * b) \supseteq(\alpha(x) \cap \alpha(a)) \cap(\alpha(y) \cap \alpha(b))=(\alpha(x) \\
& \cap \alpha(y)) \cap(\alpha(a) \cap \alpha(b))=\alpha_{E \wedge F}(x, y) \cap \alpha_{E \wedge F}(a, b),
\end{aligned}
$$

Hence, $\left(<\alpha_{E \wedge F}, \beta_{E \wedge F}>, E \times F\right)$ is a double-framed soft algebra over $\mathrm{U}$.

\section{Conclusion}

We have introduced the notion of double-framed soft algebras in a BF-algebras. We have considered the characterization of double-framed soft algebra. We have shown that every double-framed soft subset of a doubleframed soft algebra is also a double-framed soft algebra, the double-framed soft int-uni set of two double-framed soft algebras is a double- framed soft algebra. Finally the $\left(\alpha_{\wedge}, \beta_{v}\right)$-product of double-framed soft algebras $(<\alpha, \beta>, E)$ and $(<\alpha, \beta>, F)$ is also a double-framed soft algebra.

Given a double-framed soft algebra, we have made a new double-framed soft algebra.

Future research will focus on applying the results of this paper to the related algebraic structures.

\section{Acknowledgement}

The authors would like to express their thanks to the referees for their comments and suggestions which improved the paper.

\section{References}

1. Molodtsov D. Soft set theory-first results. Comput Math Appl. 1999; 37(4/5):19-31.

2. Maji PK, Roy AR, Biswas R. An application of soft sets in a decision making problem. Comput Math Appl. 2002; 44 (8-9):1077-83.

3. Maji PK, Bismas R, Roy AR. Soft set theory. Comput Math Appl. 2003; 45(4-5):555-62.

4. Maji PK, Biswas R, Roy R. Fuzzy soft sets. J of Fuzzy Math. 2001; 9(3):589-602.

5. Imai Y, Iseki K. On axiom systems of propositional calculi, XIV. Proc. Japan Acad. 1966; 42(1):19-22.

6. Iseki K, Tanaka S. An introduction to the theory of BCKalgebras. Math Japanica. 1978; 23(1):1-26.

7. Neggers J, Kim HS. On B-algebras. Math. Vensik. 2002; 54(1-2):21-9.

8. Walendziak A. On BF-algebras. Mathematica Slovaca. 2007; 57(2):119-28.

9. $\mathrm{Hu} \mathrm{QP}, \mathrm{Li} \mathrm{X}$. On proper BCH-algebras. Math Japanica. 1985; 30(1):659-61.

10. Aktaş H, Çağman N. Soft sets and soft groups. Inform Sci. 2007; 177(13):2726-35.

11. Hu QP, Li X. On BCH-algebras. Mathematics Seminar Notes. 1983; 11(1):313-20. 